\title{
Pilonidal Cyst
}

National Cancer Institute

\section{Source}

National Cancer Institute. Pilonidal Cyst. NCI Thesaurus. Code C34931.

An abnormal, fluid-filled pocket of skin that is usually located in the natal cleft, and which also often contains hair and skin debris. 